\title{
EXTENSION SETS FOR REAL ANALYTIC FUNCTIONS AND APPLICATIONS TO RADON TRANSFORMS
}

\author{
VERNOR ARGUEDAS \\ and \\ RICARDO ESTRADA
}

Escuela de Matemática

Universidad de Costa Rica

San José, COSTA RICA

(Received November 23, 1994)

\begin{abstract}
The real analytic character of a function $f(x, y)$ is determined from its behavior along radial directions $f_{\theta}(s)=f(s \cos \theta, s \sin \theta)$ for $\theta \in E$, where $E$ is a "small" set A support theorem for Radon transforms in the plane is proved In particular if $f_{\theta}$ extends to an entire function for $\theta \in E$ and $f(x, y)$ is real analytic in $\mathbb{R}^{2}$ then it also extends to an entire function in $\mathbb{C}^{2}$
\end{abstract}

KEY WORDS AND PHRASES: Real-analytic functions, real analytic functions of exponential type, Radon transform

1991 AMS SUBJECT CLASSIFICATION CODES: 42A05, 44A12

\section{INTRODUCTION}

The determination of the behavior of functions on small sets is an old problem that has been studied in many branches of analysis The purpose of this article is to study the real-analytic character of a function of two variables $f(x, y)$ from its behavior along radial directions $f_{\theta}(s)=f(s \cos \theta, s \sin \theta)$ for $\theta \in E$, where $E$ is a "small" set

We introduce a property of sets, the separation condition, that allow us to infer the behavior of $f(x, y)$ from that of $f_{\theta}(s)$ for $\theta \in E$. In particular, if $f_{\theta}$ extends to an entire function for each $\theta \in E$ and if $f(x, y)$ is real analytic in $\mathbb{R}^{2}$ then it also extends to an entire function in $\mathbb{C}^{2}$ If $f_{\theta}$ is of exponential order for $\theta \in E$ then so is $f$

The plan of the paper is as follows The second section gives the definition of the separation condition and studies some of its properties The third section gives the main results on the characterization of the real analytic character of $f(x, y)$ from that of $f_{\theta}(s)$ for $\theta \in E$, a set that satisfies the separation condition The last section applies these results to obtain a support theorem for Radon transforms in the plane

\section{THE SEPARATION CONDITION}

Motivated by its prospective use in the theory of Radon transforms, we introduce the following definition

DEFINITION. Let $\epsilon>0$ We say that a set $A \subseteq \mathbb{R}$ satisfies the separation condition of order $\epsilon$, denoted as $\mathbf{S} \mathbf{C}(\epsilon)$, if for each $n \in \mathbb{N}$ there are points $\theta_{1}, \ldots, \theta_{n} \in A$ such that

$$
\left|\theta_{\imath}-\theta_{\jmath}\right| \geq \epsilon|i-\jmath| / n \text {. }
$$


THEOREM 1. Let $A \subseteq \mathbb{R}$ be a bounded set Then

$$
\mu(\bar{A})=\sup \{\epsilon: A \text { satisfies S C }(\epsilon)\},
$$

where $\mu$ is the Lebesgue measure In particular, $A$ satisfies $\mathrm{S} \mathrm{C}$ if and only if $\mu(\bar{A})>0$

PROOF. Let $\epsilon<\mu(\bar{A})$ and let $\delta$ be such that $\epsilon<\delta<\mu(\bar{A})$ Let $\theta_{1} \in \bar{A}$ with $\mu\left(\bar{A} \cap\left[\theta_{1}, \infty[) \geq \delta\right.\right.$ and define $\theta_{2}, \ldots, \theta_{n} \in A$ recursively as

$$
\theta_{k+1}=\inf \left\{\theta \in \bar{A}: \mu\left(\bar{A} \cap\left[\theta_{k}, \theta\right]\right) \geq \delta / n\right\} .
$$

Then $\theta_{k+1}-\theta_{k} \geq \delta / n, 1 \leq k \leq n-1$ Let now $\lambda_{1}, \ldots, \lambda_{n} \in A$ be chosen so that

$$
\left|\theta_{k}-\lambda_{k}\right|<(\delta-\epsilon) / 2 n \text {. }
$$

Then $\lambda_{k+1}-\lambda_{k} \geq \epsilon / n$ It follows that $A$ satisfies S C $(\epsilon)$ and, consequently,

$$
\mu(\bar{A}) \leq \sup \{\epsilon \text { : satisfies S C. }(\epsilon)\} .
$$

Conversely, suppose $A$ satisfies S C $(\epsilon)$ Let $I$ be the closed interval $[\inf A, \sup A]$ Then $I \backslash \bar{A}$ is open, therefore

$$
I \backslash \bar{A}=\bigcup_{\jmath=1}^{\infty} \cup_{\jmath},
$$

where the $\cup_{\jmath}$ are disjoint open intervals For each $q=1,2,3, \ldots$ the set

$$
I \backslash \bigcup_{j=1}^{q} \cup_{j}
$$

consists of $q+1$ disjoint closed intervals $A_{1}^{(q)}, \ldots, A_{q+1}^{(q)}$, ordered from left to right Since $A$ satisfies S C $(\epsilon)$, there exists $\theta_{1}, \ldots, \theta_{n} \in A$, with $\theta_{1}<\theta_{2}<\ldots<\theta_{n}$, such that

$$
\left|\theta_{2}-\theta_{\jmath}\right| \geq|i-j| \epsilon / n
$$

Then there are integers, $1=k_{1} \leq k_{2} \leq \ldots \leq k_{q+1} \leq k_{q+2}=n+2$, such that $\theta_{2} \in A_{\jmath}^{(q)}$ if $k_{\jmath} \leq i<k_{\jmath+1}$

Thus

$$
\begin{aligned}
\sum_{\jmath=1}^{q+1} \mu\left(A_{\jmath}^{(q)}\right) & \geq \sum_{\jmath=1}^{q+1}\left(\theta_{k_{\jmath+1}-1}-\theta_{k_{\jmath}}\right) \\
& \geq \frac{\epsilon}{n} \sum_{\jmath=1}^{q+1}\left(k_{\jmath+1}-k_{\jmath}-1\right) \\
& \geq \frac{\epsilon}{n}(n-q) .
\end{aligned}
$$

If we first let $n \rightarrow \infty$ we obtain

$$
\epsilon \leq \sum_{\jmath=1}^{q+1} \mu\left(A_{\jmath}^{(q)}\right)=\mu\left(I \backslash \bigcup_{\jmath=1}^{q} \cup_{\jmath}\right)
$$

and if we then let $q \rightarrow \infty$ we obtain

$$
\epsilon \leq \mu(\bar{A})
$$

as desired

Let now $E$ be a subset of the circle $S=\{z \in \mathbb{C}:|z|=1\}$ We assume $E$ symmetric, namely, $-\theta \in E$ if $\theta \in E$ We denote by $\widehat{E}$ the set of arguments of elements of $E$, so that $E=\left\{e^{\imath \theta}: \theta \in \widehat{E}\right\}$ 
DEFINITION. We say that $E$ satisfies the separation condition of order $\epsilon$ (for symmetric sets), denoted as $\mathrm{S} \mathrm{C}(\epsilon)$, if for each $n \in \mathbb{N}$ there are arguments $\theta_{1}, \ldots, \theta_{n} \in \widehat{E}$ such that

$$
\left|\theta_{2}-\theta_{\jmath}+k \pi\right| \geq \frac{\epsilon|\imath-\jmath|}{n},
$$

for each $k \in \mathbb{Z}$

Notice that the separation condition for not necessarily symmetric sets requires (26) to hold for $k$ even only In our case, however, the points $e^{\imath \theta}$ and $-e^{\imath(\theta+\pi)}$ will be identified and that is why we require (26) for all $k \in \mathbb{Z}$

It is not hard to see that $E$ satisfies S C $(\epsilon)$ if and only if $\widehat{E} \cap[\alpha, \alpha+\pi]$ satisfies S C $(\epsilon)$ for some, and hence for each, $\alpha \in \mathbb{R}$ It follows that

$$
\sup \{\epsilon: \text { satisfies S C }(\epsilon)\}=\frac{\mu(\bar{E})}{2},
$$

so that, in particular, $E$ satisfies S C if and only if $\mu(\bar{E})>0$.

\section{EXTENSION OF REAL ANALYTIC FUNCTIONS}

Let $E$ be a symmetric subset of the unit circle We denoted by $\Omega_{E, r}$ and $\Omega_{E}$ the sectors

$$
\begin{gathered}
\Omega_{E, r}=\{s z: 0 \leq s \leq r, z \in E\}, \\
\Omega_{E}=\bigcup_{r>0} \Omega_{E, r}=\{s z: 0 \leq s, z \in E\} .
\end{gathered}
$$

Let $X_{E, r}$ be the space of germs of functions $f$ defined in $\Omega_{E, r}$, that satisfy the following conditions

a) $f$ can be extended to a neighborhood of the origin as a smooth (i e $C^{\infty}$ ) function,

b) $f_{\theta}(s)=f(s \cos \theta, s \sin \theta)$ is real-analytic as a function of $s$ for $|s|<r$ for each $\theta \in \widehat{E}$,

c) the quantity

$$
\|f\|_{E, r}=\sup \left\{\sum_{n=0}^{\infty} \frac{\left|f_{\theta}^{(n)}(0)\right| r^{n}}{n !}: \theta \in \widehat{E}\right\}
$$

is finite

Observe that if the functions $f_{1}, f_{2}$ extend to smooth functions $F_{1}, F_{2}$ that agree of infinite order at the origin, then they are identified

The space $X_{E}$ is defined as the intersection of the $X_{E, r}$ for $r>0$ Observe that if $f \in X_{E}$ then $f_{\theta}(s)$ can be extended to an entire function of $s \in \mathbb{C}$ for each $\theta \in \widehat{E}$

Let $F \subseteq S$ be a superset of $E, E \subset F$ Then each element of $X_{F, r}$ can be restricted to $X_{E, r}$ Our aim is to show that under suitable assumptions on $E$ this process can be reversed, namely, each element of $X_{E, r}$ can be extended to $X_{F, t}$ for some $t \leq r$ In particular, $X_{E}$ and $X_{F}$ would result to be isomorphic and, by taking $F=S$, each element of $X_{E}$ would extend to an entire function in $\mathbb{C}^{2}$

Let us first show how to compute the directional derivatives $f_{\lambda}^{(n)}(0)$ from the corresponding derivative values $f_{\theta}^{(n)}(0)$ for $\theta \in E$ Indeed, let us first suppose $\lambda=0$ Then for each $\theta \in E$ we have

$$
f_{\theta}^{(n)}(0)=\sum_{k=0}^{n}\left(\begin{array}{l}
n \\
k
\end{array}\right) \cos ^{k} \theta \sin ^{n-k} \theta \frac{\partial^{n} f(\mathbf{0})}{\partial x^{k} \partial y^{n-k}} .
$$

If $\theta_{0}, \theta_{1}, \ldots, \theta_{n} \in E$ then evaluating ( 34 ) at $\theta=\theta_{2}$ yields a linear system for the computation of the partial derivatives 


$$
\frac{\partial^{n} f(\mathbf{0})}{\partial x^{k} \partial y^{n-k}}, \quad 0 \leq k \leq n
$$

Solving for

$$
\frac{\partial^{n} f}{\partial x^{n}}(\mathbf{0})=f_{0}^{(n)}(0)
$$

gives

$$
f_{0}^{(n)}(0)=\sum_{\imath=0}^{n} f_{\theta_{1}}^{(n)}(0) \frac{\triangle_{n-1}\left(\theta_{0}, \ldots, \theta_{2-1}, \theta_{\imath+1}, \ldots, \theta_{n}\right) \Pi_{j \neq \imath} \sin \theta_{j}}{\triangle_{n}\left(\theta_{0}, \ldots, \theta_{n}\right)}
$$

where we have denoted by $\triangle_{n}\left(\theta_{0}, \ldots, \theta_{n}\right)$ the determinant

$$
\triangle_{n}\left(\theta_{0}, \ldots, \theta_{n}\right)=\operatorname{det}\left[\cos ^{k} \theta_{2} \sin ^{n-k} \theta_{2}\right]_{0 \leq k, 2 \leq n} .
$$

These determinants are easily evaluated if we observe that

$$
\begin{gathered}
\triangle_{n}\left(0, \theta_{1}, \ldots, \theta_{n}\right)=\triangle_{n-1}\left(\theta_{1}, \ldots, \theta_{n}\right) \sin \theta_{1} \ldots \sin \theta_{n}, \\
\triangle_{n}\left(\theta_{0}, \ldots, \theta_{n}\right)=\triangle_{n}\left(\theta_{0}+\theta, \ldots, \theta_{n}+\theta\right), \\
\triangle_{1}\left(\theta_{0}, \theta_{1}\right)=\sin \left(\theta_{0}-\theta_{1}\right) .
\end{gathered}
$$

This yields

$$
\triangle_{n}\left(\theta_{0}, \ldots, \theta_{n}\right)=\prod_{\imath<j} \sin \left(\theta_{\imath}-\theta_{\jmath}\right)
$$

Replacing 0 by $\theta$, using ( 310$)$ and doing some simplification in (3 5) give the formula

$$
f_{\theta}^{(n)}(0)=\sum_{i=0}^{n} f_{\theta_{1}}(0) \prod_{\jmath \neq \imath} \frac{\sin \left(\theta-\theta_{\jmath}\right)}{\sin \left(\theta_{2}-\theta_{\jmath}\right)}
$$

Naturally (3 11) can be applied only if $\triangle_{n}\left(\theta_{0}, \ldots, \theta_{n}\right) \neq 0$, that is, only if $\theta_{\imath}-\theta_{\jmath} \neq k \pi$ for $i \neq \jmath$ and $k \in \mathbb{Z}$ When $E$ satisfies the S $\mathrm{C}$ then such points $\theta_{0}, \ldots, \theta_{1}$ can always be found, but, more than that, if they satisfy the separation condition

$$
\left|\theta_{2}-\theta_{\jmath}+k \pi\right| \geq \frac{|i-\jmath| \epsilon}{n+1}, \quad 0 \leq i, \quad j \leq n,
$$

then $f_{\theta}^{(n)}(0)$ can be estimated on $S \backslash E$ from estimates on $E$. Actually, if we suppose that $\left|f_{\theta}^{(n)}\right| \leq b_{n}$ for each $\theta \in \widehat{E}$ then (3 11) yields

$$
\left|f_{\theta}^{(n)}(0)\right| \leq b_{n} M(\theta)^{n} \sum_{i=0}^{n} \prod_{\jmath \neq \imath}\left|\sin \left(\theta_{\imath}-\theta_{\jmath}\right)\right|^{-1},
$$

where

$$
M(\theta)=\sup \{|\sin (\theta-\psi)|: \psi \in \widehat{E}\} \leq 1 .
$$

But $\left|\frac{\sin x}{x}\right| \geq 2 / \pi$ for $0<|x| \leq \pi / 2$, so that if for each $i, j$ we choose $k=k(i, \jmath) \in \mathbb{Z}$ with $\left|\theta_{2}-\theta_{\jmath}+k \pi\right| \leq \pi / 2$ then we obtain 


$$
\begin{aligned}
\prod_{\jmath \neq 2}\left|\sin \left(\theta_{2}-\theta_{\jmath}\right)\right| & \geq\left(\frac{2}{\pi}\right)^{n} \prod_{j \neq \imath}\left|\theta_{\imath}-\theta_{\jmath}+k \pi\right| \\
& \geq\left(\frac{2}{\pi}\right)^{n} \prod_{\jmath \neq \imath}\left\{\frac{|i-\jmath| \epsilon}{n+1}\right\} \\
& \geq\left[\frac{2 \epsilon}{\pi(n+1)}\right]^{n}(n-i) ! \imath !
\end{aligned}
$$

so that

$$
\begin{aligned}
\left|f_{\theta}^{(n)}(0)\right| & \leq b_{n} M(\theta)^{n} \sum_{i=0}^{n}\left[\frac{\pi(n+1)}{2 \epsilon}\right]^{n} \frac{1}{(n-1) ! i !} \\
& \leq \frac{b_{n}}{n !}\left[\frac{\pi M(\theta)(n+1)}{\epsilon}\right]^{n} .
\end{aligned}
$$

Specializing the value of $b_{n}$ in (3 16) we obtain several extension theorems

THEOREM 2. If $E$ satisfies the $\mathrm{S} \mathrm{C}(\epsilon)$ then each $f \in X_{E, r}$ can be extended to $X_{E, t}$ for each $F \supseteq E$ whenever $t<\epsilon r / M \pi e$, where $M=\sup \{M(\theta): \theta \in(\widehat{S \backslash E)}\}$

PROOF. If $f \in X_{E, r}$ then according to (3 3)

$$
\left|f_{\theta}^{(n)}(0)\right| \leq \frac{n ! K}{r^{n}}
$$

where $K=\|f\|_{E, r} \quad$ Thus for each $\theta \in \mathbb{R}$ the series

$$
\sum_{n=0}^{\infty} \frac{f_{\theta}^{(n)}(0)}{n !} s^{n}
$$

is majorized by the series

$$
\sum_{n=0}^{\infty} \frac{K}{n !}\left[\frac{M(n+1) \pi s}{r \epsilon}\right]^{n}
$$

which converges for $s \leq \epsilon r / M \pi e$

THEOREM 3. If $E$ satisfies the S C $(\epsilon)$ then $X_{E}$ is isomorphic to $X_{F}$ for each $F \supseteq E$. If $f \in X_{E}$ then the Taylor series

$$
\sum_{k_{1}=0}^{\infty} \sum_{k_{2}=0}^{\infty} \frac{\partial^{k_{1}+k_{2}}}{\partial x^{k_{1}} \partial y^{k_{2}}} f(0,0) \frac{z_{1}^{k_{1}} z_{2}^{k_{2}}}{k_{1} ! k_{2} !}
$$

defines an entire function in $\mathbb{C}^{2}$

PROOF. That $X_{E}$ is isomorphic to $X_{F}$ if $F \supset E$ follows by letting $r \rightarrow \infty$ in the previous theorem The convergence of the Taylor series

$$
\sum_{k_{1}=0}^{\infty} \sum_{k_{2}=0}^{\infty} \frac{\partial^{k_{1}+k_{2}}}{\partial x^{k_{1}} \partial y^{k_{2}}} f(0,0) \frac{z_{1}^{k_{1}} z_{2}^{k_{2}}}{k_{1} ! k_{2} !}
$$

for each $\left(z_{1}, z_{2}\right) \in \mathbb{C}^{2}$ with $\left|z_{1}\right|<r,\left|z_{2}\right|<r$ is equivalent to the convergence of the series

$$
\sum_{k=0}^{\infty} \frac{f_{\theta}^{(k)}(0)}{k !} z^{n}
$$

for each $|z|<r$ and each $\theta \in \mathbb{R}$, so that the Taylor series indeed defines an entire function in $\mathbb{C}^{2}$

A particularly useful result along these lines is the following 
COROLlaRY. Let $f$ be real analytic in $\mathbb{R}^{2}$ If $f \in X_{E}$ for some $E$ that satisfies the S C then $f$ extends to an entire function in $\mathbb{C}^{2}$ and its Taylor series converges to $f$ in all $\mathbb{R}^{2}$

We say that an entire function of $n$ variables $F\left(z_{1}, \ldots, z_{n}\right)$ is of exponential order if there are constants $K$ and $A$ such that

$$
\left|F\left(z_{1}, \ldots, z_{n}\right)\right| \leq K e^{A\left(\left|z_{1}\right|++\left|z_{n}\right|\right)}
$$

for each $\left(z_{1}, \ldots, z_{n}\right) \in \mathbb{C}^{n}$ In that case we say that $F$ is of exponential order $A$

THEOREM 4. Let $f(x, y)$ be real analytic in $\mathbb{R}^{2}$ Let $f \in X_{E}$, where $E$ satisfies the S C $(\epsilon)$ If $f_{\theta}(z)=f(z \cos \theta, z \sin \theta)$ is of uniform exponential order in $E$ in the sense that

$$
\left|f_{\theta}(z)\right| \leq K e^{A|z|}, \quad z \in \mathbb{C}, \quad \theta \in \widehat{E},
$$

then $f$ extends to an entire function of exponential order in $\mathbb{C}^{2}$

PROOF. If $f_{\theta}(z)$ satisfies $(319)$ then it follows by Cauchy estimates that

$$
\left|f_{\theta}^{(n)}(0)\right| \leq K n !\left(\frac{A e}{n}\right)^{n}, \quad \theta \in \widehat{E}
$$

If we now use ( 316$)$ we obtain the bound

$$
\left|f_{\theta}^{(n)}(0)\right| \leq K\left[\frac{A \pi e(n+1) M(\theta)}{\epsilon n}\right]^{n} \leq K \epsilon\left[\frac{A \pi e M(\theta)}{\epsilon}\right]^{n} .
$$

Up to now we have suppose $\theta$ real, but as should be clear (3 21) also holds in $\theta \in \mathbb{C}$ If $\theta \in \mathbb{R}$ then $|M(\theta)| \leq 1$, an inequality that has to be replaced by

$$
|M(\theta)| \leq|\cos \theta|+|\sin \theta|
$$

if $\theta \in \mathbb{C}$

It follows that if $z_{1}=w \cos \theta, z_{2}=w \sin \theta$ for some $w, \theta \in \mathbb{C}$ then

$$
\left|f\left(z_{1}, z_{2}\right)\right|=\left|f_{\theta}(w)\right| \leq K e \sum_{n=0}^{\infty} \frac{1}{n !}\left[\frac{A \pi e M(\theta)|w|}{\epsilon}\right]^{n} \leq K e e^{A \pi e M(\theta)|w| e^{-1}}
$$

or

$$
\left|f\left(z_{1}, z_{2}\right)\right| \leq K e^{1+(A \pi e / \epsilon)\left(\left|z_{1}\right|+\left|z_{2}\right|\right)} .
$$

Not every pair $\left(z_{1}, z_{2}\right) \in \mathbb{C}^{2}$ admits the representation $z_{1}=w \cos \theta, z_{2}=w \sin \theta$ the extra condition $z_{1}^{2}+z_{2}^{2} \neq 0$ has to be satisfied However, the set $\left\{\left(z_{1}, z_{2}\right): z_{1}^{2}+z_{2}^{2} \neq 0\right\}$ is dense in $\mathbb{C}^{2}$, so that by continuity (3.24) holds in all $\mathbb{C}^{2}$

REMARKS.

1) Notice that our definition of $X_{E, r}$ requires $f$ to have a smooth extension to a neighborhood of the origin. A function like $f(x, y)=\left(x^{2}+y^{2}\right)^{2}\left(x^{2}+2 y^{2}\right)^{-1}$ shows that our theorems do not hold if condition (a) is omitted in the definition of $X_{E, r}$

2) A function like

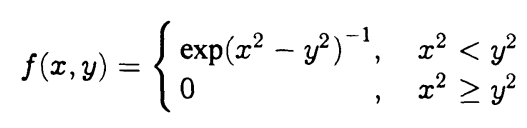

shows that Theorem 4 is false if $f$ is not required to be real analytic but just smooth

\section{APPLICATION TO RADON TRANSFORMS}

In this section we show that our results have application in the support theorems for Radon transforms 
If $f$ is a function defined in $\mathbb{R}^{n}$, integrable over every hyperplane, then its Radon transform is defined as

$$
\widehat{f}(\mathbf{w}, t)=\int_{\mathbf{x} \cdot \mathbf{w}-t} f(\mathbf{x}) d \mu(\mathbf{x})
$$

for $(\mathbf{w}, t) \in S^{n-1} \times \mathbb{R}$, where $d \mu$ is the Lebesgue measure on the hyperplane $\mathbf{x} \cdot \mathbf{w}=t$

Clearly, if $f$ has compact support contained in the closed ball $\overline{B(\mathbf{0}, A)}$ then the support of its Radon transform $\widehat{f}$ is contained in $s^{n-1} \times[-A, A]$ The converse is not true in general $[6,11]$, unless $f$ satisfies some additional conditions For instance, the support theorem of Helgason $[4,5,8]$ says that if $f$ is continuous and rapidly decreasing (i e $f(\mathbf{x})=0\left(|\mathbf{x}|^{-k}\right.$ ) as $|\mathbf{x}| \rightarrow \infty$ ) then the support of $\widehat{f}$ is contained in $S^{n-1} \times[-A, A]$ if and only if the support of $f$ is contained in $B(\mathbf{0}, A)$ In [10], Wiegernnck introduced a simple but powerful method for the study of support theorems As we show, the use of this method and of our results of the previous sections allow us to obtain interesting support results for the Radon transform

Instead of requiring $f$ to be rapidly decreasing in the ordinary sense, it would be enough to ask $f$ to be distributionally small $[1]$ in the sense that it satisfies the moment asymptotic expansion $[2,3]$

$$
f(\lambda \mathbf{x}) \sim \sum_{|\mathbf{k}|=0}^{\infty} \frac{(-1)^{|\mathbf{k}|} \mu_{\mathbf{k}} \mathbf{D}^{\mathbf{k}} \delta(\mathbf{x})}{\mathbf{k} ! \lambda^{|\mathbf{k}|+n}}, \quad \text { as } \quad \lambda \rightarrow \infty ; \quad \mu_{\mathbf{k}}=\left\langle f(\mathbf{x}), \mathbf{x}^{\mathbf{k}}\right\rangle .
$$

For our purposes, it would be convenient to take $f \in \mathcal{O}_{C}^{\prime}$ The space $\mathcal{O}_{C}$ (see [7,9]) is defined as the space of smooth functions $\phi(\mathbf{x})$ defined in $\mathbb{R}^{n}$ that satisfy $\mathbf{D}^{\mathbf{k}} \phi(\mathbf{x})=0\left(|\mathbf{x}|^{q}\right)$ as $|\mathbf{x}| \rightarrow \infty$ for each $\mathbf{k} \in \mathbb{N}^{n}$ and some $q \in \mathbb{R} \quad$ The space $\mathcal{O}_{M}$ is defined similarly but now $\mathbf{D}^{\mathbf{k}} \phi(\mathbf{x})=0\left(|\mathbf{x}|^{q(\mathbf{k})}\right)$ for some $q(\mathbf{k})$ that may vary with $\mathbf{k}$ The Fourier transform interchanges these spaces $\mathcal{F}\left(\mathcal{O}_{C}^{\prime}\right)=\mathcal{O}_{M}, \mathcal{F}\left(\mathcal{O}_{M}^{\prime}\right)=\mathcal{O}_{C}$ Every element of $\mathcal{O}_{C}^{\prime}$ is distributionally small [2,3] Also observe that if $f \in \mathcal{O}_{C}^{\prime}$ then its Fourier transform $\mathcal{F}\{f(\mathbf{x}), \mathbf{u}\}=\left\langle f(\mathbf{x}), e^{2 \mathbf{x} \cdot \mathbf{u}}\right\rangle$ can be obtained by evaluation at $e^{2 \mathbf{x} \cdot \mathbf{u}}$, not just duality

THEOREM 5. Let $f$ be a continuous function in $\mathbb{R}^{2}$ that satisfies three conditions

(a) $f \in \mathcal{O}_{C}^{\prime}$

(b) Its Radon transform satisfies the estimate

$$
|\widehat{f}(\mathbf{w}, t)| \leq C_{\mathbf{w}} e^{-\epsilon_{\mathbf{w}}|t|}, \quad \epsilon_{\mathbf{w}}>0, \quad(\mathbf{w}, t) \in S \times \mathbb{R} .
$$

(c) There is a set $E \subseteq S$ that satisfies the $\mathrm{S} \mathrm{C}(\epsilon)$ and a number $R>0$ such that

$$
\widehat{f}(\mathbf{w}, t)=0, \quad \mathbf{w} \in E, \quad|t|>R .
$$

Then $f$ has compact support contained in the disc $\overline{B(\mathbf{0}, R \pi e / \epsilon)}$

PROOF. If $f \in \mathcal{O}_{C}^{\prime}$ then its Fourier transform $F=\mathcal{F}(f)$ belongs to $\mathcal{O}_{M}$, so that $F$ is smooth in $\mathbb{R}^{2}$ If $|\mathbf{w}|=1$, the values of $F$ along the line $s \mathbf{w}, s \in \mathbb{R}$, can be expressed in terms of the Radon transform as

$$
F(s \mathbf{w})=\int_{\mathbb{R}^{2}} f(\mathbf{x}) e^{\imath s \mathbf{w} \cdot \mathbf{x}} d \mathbf{x}=\int_{-\infty}^{\infty} \widehat{f}(\mathbf{w}, t) e^{-\imath s t} d t .
$$

The condition (b) guarantees that $F(s \mathbf{w})$ can be extended to strip $\left\{s \in \mathbb{C}:|\operatorname{Im} s|<\epsilon_{\mathbf{w}}\right\}$ so that, in particular, $F(s \mathbf{w})$ is real analytic in $s$ When $\mathbf{w} \in E$ then (44) shows that $F(s \mathbf{w})$ extends to an entire function of exponential order $R$

$$
|F(s \mathbf{w})| \leq K e^{R|s|}, \quad s \in \mathbb{C}, \quad \mathbf{w} \in E .
$$

Using Theorem 4 it follows the Taylor series 


$$
\sum_{k_{1}=0}^{\infty} \sum_{k_{2}=0}^{\infty} \frac{1}{k_{1} ! \mid k_{2} !} \frac{\partial^{k_{1}+k_{2}} F(0,0)}{\partial x^{k_{1}} \partial y^{k_{2}}} z_{1}^{k_{1}} z_{2}^{k_{2}}
$$

defines an entire function of exponential order $R \pi e / \epsilon$ in $\mathbb{C}^{2} \quad$ But since $F$ is smooth near the origin and since $F(s \mathbf{w})$ is real analytic in $s$, it follows that the Taylor series converges to $F$ in $\mathbb{R}^{2}$ Thus $F$ extends to an entire function of exponential order $R \pi e / \epsilon$ Since $F \in \mathcal{O}_{M}$, the Paley-Wiener theory shows that $f=\mathcal{F}^{-1} F$ is a distribution with compact support in the $\operatorname{disc} \overline{B(\mathbf{0}, R \pi e / \epsilon)}$

\section{REFERENCES}

[1] ESTRADA, R, The asymptotic expansion of certain series considered by Ramanujan, Appl. Anal. 43 (1992), 191-228

[2] ESTRADA, R and KANWAL, R P , A distributional theory of asymptotic expansions, Proc. Roc. Soc. London, A428 (1990), 399-430

[3] ESTRADA, R and KANWAL, R P, The asymptotic expansion of some multidimensional generalized function, J. Math. Anal. Appls. 163 (1992), 264-283

[4] HELGASON, S, A duality in integral geometry, some generalizations of the Radon transform, Bull. Amer. Math. Soc. 70 (1964), 435-446.

[5] HELGASON, S, The Radon transform on Euclidean spaces, compact two-point homogeneous spaces and Grassmann manifolds, Act. Math. 113 (1965), 153-180

[6] HELGASON, S, The Radon Transform, Birkhauser, Boston, 1980

[7] HORVATH, J, Topological Vector Spaces and Distributions, Vol I, Addison-Wesley, Reading, 1966

[8] LUDWIG, D, The Radon transform on Euclidean space, Comm. Pure Appl. Math. 23 (1966), 4981

[9] SCHWARTZ, L, Théorie des Distributions, Hermann, Paris, 1966.

[10] WIEGERINK, J J O O, A support theorem for Radon transforms on $\mathbb{R}^{n}$, Proc. Koninklije Neder. Akad. Wettensch. A88 (1985), 87-93

[11] ZALCMAN, L, Uniqueness and non-uniqueness for the Radon transform, Bull. London Math. Soc. 14 (1982), 241-245 


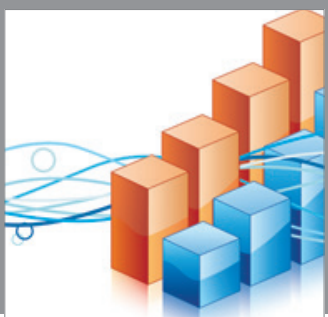

Advances in

Operations Research

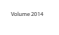

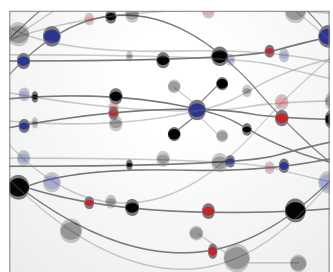

\section{The Scientific} World Journal
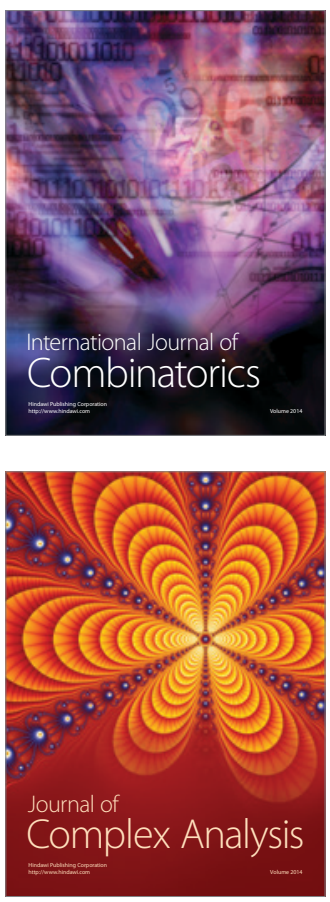

International Journal of

Mathematics and

Mathematical

Sciences
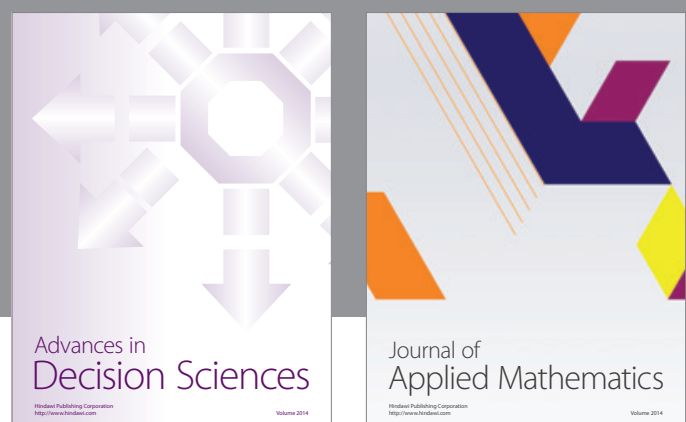

Journal of

Applied Mathematics
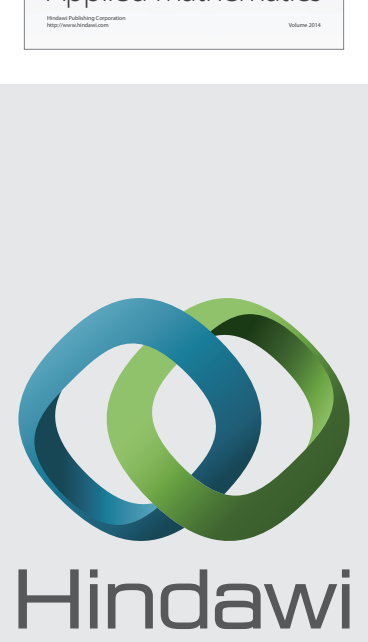

Submit your manuscripts at http://www.hindawi.com
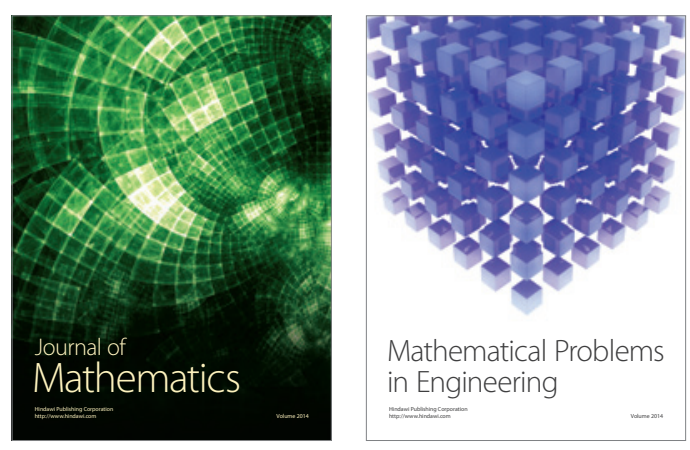

Mathematical Problems in Engineering
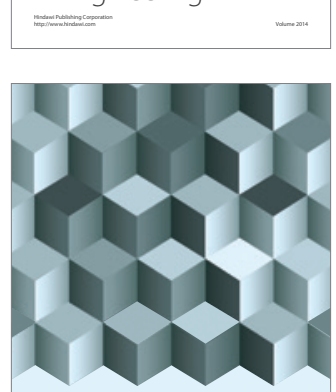

Journal of

Function Spaces
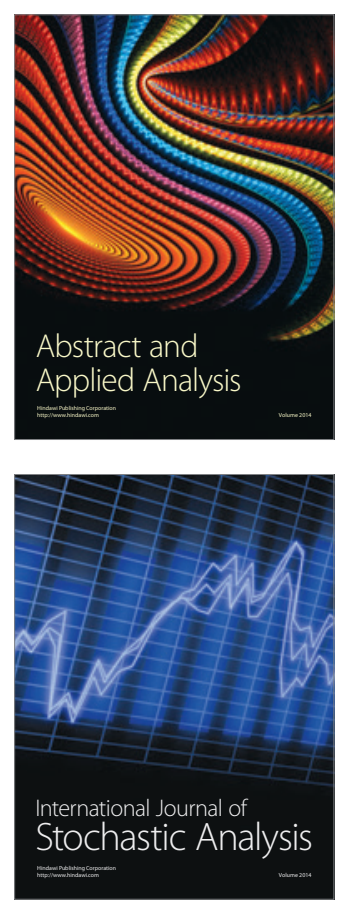

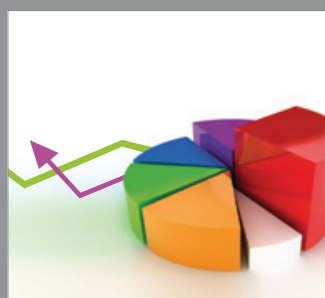

ournal of

Probability and Statistics

Promensencen
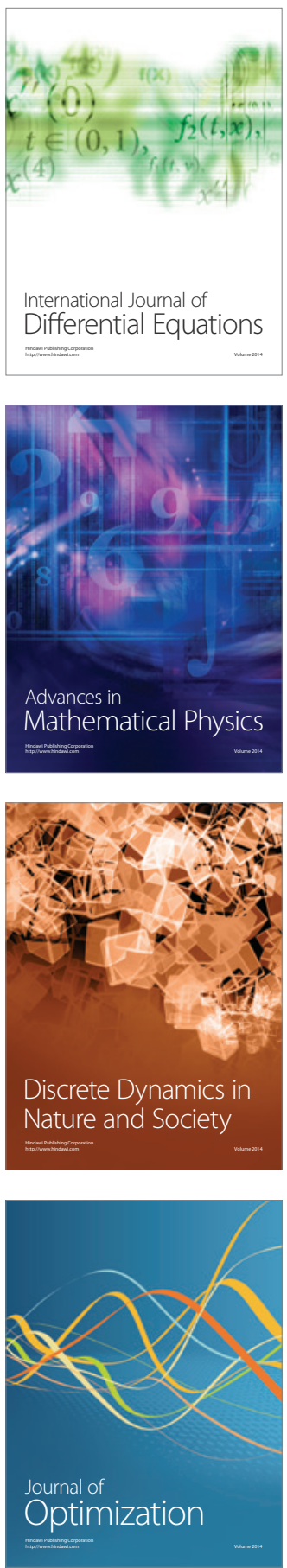